\title{
Cost Function based Soft Feedback Iterative Channel Estimation in OFDM Underwater Acoustic Communication
}

\author{
Gang QIAO, Zeeshan Babar, Lu Ma and Xue LI
}

\begin{abstract}
Underwater Acoustic (UWA) communication is mainly characterized by bandwidth limited complex UWA channels. Orthogonal Frequency Division Multiplexing (OFDM) solves the bandwidth problem and an efficient channel estimation scheme estimates the channel parameters. Iterative channel estimation refines the channel estimation by reducing the number of pilots and coupling the channel estimator with channel decoder. This paper proposes an iterative receiver for OFDM UWA communication, based on a novel cost function threshold driven soft decision feedback iterative channel estimation technique. The receiver exploits orthogonal matching pursuit (OMP) channel estimation and low density parity check (LDPC) coding techniques after comparing different channel estimation and coding schemes. The performance of the proposed receiver is verified by simulations as well as sea experiments. Furthermore, the proposed iterative receiver is compared with other noniterative and soft decision feedback iterative receivers.
\end{abstract}

Index Terms - Channel Estimation, Equalization, Iterative Receiver, OFDM, Underwater Communication.

\section{INTRODUCTION}

$\mathrm{U}$ NDERWATER Acoustic (UWA) communication is challenging because of the extremely limited bandwidth, slow speed of sound, multipath, delay spread, signal attenuation and ambient noise. The UWA channel makes it different from terrestrial communication. . Inter-symbol interference (ISI) and Inter-carrier interference (ICI) are introduced into the transmitted signal by the channel. Channel estimation estimates the channel parameters and equalization removes the effects of the channel on the received signal [1]. The role of channel estimation is of prime importance in designing any communication model. Different channel estimation schemes were applied to OFDM UWA

This work was supported partially by the National Natural Foundation of China under Grant 61431004 and Grant 61601136, and partially by the Sustainable Funding of the Key Laboratory of Underwater Acoustic Technology under Grant SSJSWDZC2018006 and the Science and Technology on Underwater Acoustic Antagonizing Laboratory Foundation.

Gang Qiao and $\mathrm{Lu} \mathrm{Ma}$ are with the College of Underwater Acoustic Engineering, Harbin Engineering University, Harbin 150001, China, and also with the Science and Technology on Underwater Acoustic Antagonizing Laboratory, Beijing, China.

Zeeshan Babar and Xue Li are with the College of Underwater Acoustic Engineering, Harbin Engineering University, Harbin 150001, China, and also with the Acoustic Science and Technology Laboratory and Key Laboratory of Marine Information Acquisition and Security, Harbin Engineering University, Harbin 150001, China (e-mail: babar_zeeshan; qiaogang; malu; xueli $@$ hrbeu.edu.cn) communication depending upon the requirement of the model [2]. Many such scheme are summarized and compared with each other in our review article previously [3]. Least Square (LS) is one of commonly used channel estimation scheme, where pilot tones are used for channel estimation [4]. In this case many subcarriers need to be assigned to pilot subcarriers and therefore the data rate is affected. To increase the efficiency, many iterative/adaptive channel estimation schemes were introduced and were proved to be more efficient as it reduces the number of pilots $[5,6]$. Furthermore the performance of the iterative channel estimation depends on the type of decision feedback used; feedback methods like hard decision and soft decision feedback methods were introduced $[7,8]$. Compressed sensing based channel estimation was introduced for sparse channels where a dictionary was used to formulate the channel coefficient vector [9]. Orthogonal Matching Pursuit is one such algorithm widely used for OFDM UWA communication [10].

Channel coding adds some redundancy in the useful bits in order to protect the data in noisy channel. Trellis Coded Modulation (TCM), convolutional codes, Reed Solomon (RS) codes, turbo codes, Space time trellis codes and low density parity check codes (LDPC) are the commonly used coding schemes used for UWA communication. LDPC code is preferred for noisy channels as its check matrix is sparse and the threshold can be set very near to Shannon capacity limit [11].

In this paper we propose an iterative receiver which exploits cost function based soft decision feedback orthogonal matching pursuit (OMP) channel estimation and LDPC coding /decoding schemes. The performance of the receiver is analyzed via simulations as well as experiments. The performance of the proposed receiver is compared with noniterative and others soft and hard decision feedback iterative receivers. Furthermore in the experimental analysis, different combinations of channel estimation techniques and coding techniques are compared using the proposed feedback method.

The rest of the paper is organized as: section 2 gives the system model, section 3 proposes the receiver design and explains cost function based soft decision feedback method, the OMP channel estimation for UWA communication and LDPC coding scheme. Section 4 gives the results including simulation and experimental results, while section 5 concludes our work. 


\section{SYSTEM AND CHANNEL MODEL}

Consider an OFDM system with symbol duration $T$ and cyclic prefix interval $T_{c p}$, so that the total OFDM block duration is $T^{\prime}=T+T_{c p}$. The subcarrier spacing is given by $\Delta f=1 / T$ and for total number of $K$ subcarriers, the bandwidth can be given by $B=K / T$. The frequency of $m$ th subcarrier is given by:

$$
f_{m}=f_{c}+m / T, \quad m=-K / 2, \ldots, K / 2-1
$$

Where $f_{c}$ denotes the center frequency. The passband signal transmitted is given by:

$$
x(t)=2 \operatorname{Re}\left\{\left[\sum_{m=-K / 2}^{m=K / 2} s[m] \mathrm{e}^{j 2 \pi f_{m} t}\right]\right\} \quad, \quad t \in[0, T]
$$

Assume that the channel is time-invariant channel within each OFDM symbol, and the channel impulse response of the multipath channel with $L$ number of paths can be given by:

$$
h(\tau)=\sum_{l=1}^{L} \xi_{l} \delta\left(\tau-\tau_{l}\right)
$$

Where $\xi_{l}$ and $\tau_{l}$ respectively denote the amplitude and delay of $l$ th path, and the received signal $\tilde{y}(t)$ with additive noise $w(t)$ can be given by:

$$
\tilde{y}(t)=\sum_{l=1}^{L} \xi_{l} \tilde{x}\left(t-\tau_{l}\right)+w(t)
$$

\section{ITERATIVE RECEIVER DESIGN}

An iterative receiver is proposed here, which uses cost function based soft decision feedback OMP channel estimation and LDPC coding /decoding algorithm as shown in Figure 1. In the preprocessing block, the value of $I$ is taken as zero, which will make the receiver similar to non-iterative receiver, where the pilot symbols will be used for channel estimation. After the decoding, the cost function based soft information will be compared with the previous iteration value, which serves as threshold and value of $I$ is incremented for next iteration. The cost function based soft decision feedback method, OMP channel estimation and LDPC decoding are explained in detail as follows.

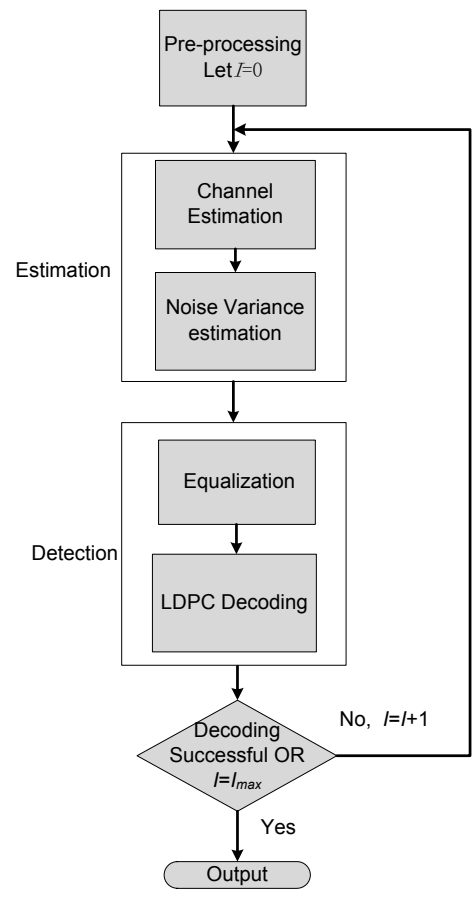

Figure 1: Iterative Receiver Block Diagram

\section{A. Cost Function Based Soft Decision Feedback}

Threshold controlled and uncontrolled soft and hard decision feedback methods are already in practice in iterative receiver systems $[12,13]$. The soft symbol estimates are claimed to be better than hard symbol estimates especially for iterative channel estimation as they provide more statistical information about the transmitted data $[14,15]$. We propose a new feedback method that uses cost function as decision condition threshold. Based on the cost function threshold, it decides whether to select the current iterative channel estimation result or to use the pilot-aided initial channel estimation value. This design is based on the idea given in detail in [15], where a cost function was used for decision feedback for coded OFDM wireless communication system. However the cost function of the channel estimation for the current iteration was always compared with the initial channel cost function $\varsigma\left(H_{m}^{(0)}\right)$, whereas we compare the cost function of the current iteration with the cost function of the previous iteration of channel estimation $\varsigma\left(\hat{H}_{m}^{(j-1)}\right)$. The advantage of this change is that the cost function keeps updating in comparison to the previous iteration, therefore the performance of channel estimation improves. Furthermore in [15], pilot-assisted channel estimation was performed based on the hard feedback method. We use soft feedback and OMP channel estimation, which obviously improves the channel estimation performance [3]. Furthermore we also compare LS channel estimation with OMP channel estimation using the same feedback method. This improved decision feedback method based on the cost function is given by: 


$$
H_{m}^{(j)}=\left\{\begin{array}{l}
\frac{Y_{m}}{\hat{X}_{m}^{(j)}} \text { if } \varsigma\left(\frac{Y_{m}}{\hat{X}_{m}^{(j)}}\right) \leq \varsigma\left(\hat{H}_{m}^{(j-1)}\right), \\
\hat{H}_{m}^{(j-1)} \text { otherwise }
\end{array},\right.
$$

where $j$ indicates the number of iterations, $\varsigma\left(\frac{Y_{m}}{\hat{X}_{m}^{(j)}}\right)$ and $\varsigma\left(\hat{H}_{m}^{(j-1)}\right)$ represents the signal detection cost function and channel estimation cost function for $m$ th subcarrier respectively. Instead of using the soft symbols directly as feedback, soft information on the reliability of $\hat{X}_{m}^{(j)}$ in the form of Log likelihood rations (LLRs) is exploited by the threshold test in equation (5). To derive the cost function $\varsigma\left(\frac{Y_{m}}{\hat{X}_{m}^{(j)}}\right)$, the iterative receiver input is taken from the demodulated received symbols, that serves as auxiliary pilots. The modified signal model at the iterative receiver is given by:

$$
Y_{m}=H_{m} \hat{X}_{m}+\eta_{m}
$$

where $\eta_{m}$ is given by:

$$
\eta_{m}=\left(X_{m}-\hat{X}_{m}\right) \cdot H_{m}+\omega_{m},
$$

The decision feedback induces this additional noise component, that can be approximated as Additive White Gaussian Noise with zero mean and its variance is given by:

$$
\sigma_{\eta_{m}}^{2}=E\left\{\left|\eta_{m}\right|^{2}\right\}=N_{0}+\sigma_{\tilde{X}_{m}}^{2} .
$$

where $N_{0}$ is the variance of AWGN signal and $\sigma_{\tilde{X}_{m}}^{2}$ can be generated from the LLR of the decoder output [15].

In the first iteration, the cost function $\varsigma\left(\frac{Y_{m}}{\hat{X}_{m}^{(j)}}\right)$ is compared with the initial cost function $\varsigma\left(\hat{H}_{m}^{(0)}\right)$, which can be derived by using the initial estimates based on pilot symbols. The channel estimation error can be given by $\boldsymbol{\varepsilon}_{m}=H_{m}-\hat{H}_{m}$. Assuming $\boldsymbol{\varepsilon}_{m}$ to be zero mean with variance equal to the mean square error, denoted by MSE $\sigma_{\mathbf{v} m}^{2}=E\left\{|\boldsymbol{\varepsilon} m|^{2}\right\}$, which depends on the FIR filter $\mathbf{v}$, therefore the variance of the effective noise term can be given by:

$$
\sigma_{\eta_{m}}^{2}=E\left\{\left|\eta_{m}\right|^{2}\right\}=N_{0}+E_{s} \sigma_{\mathbf{v}_{m}}^{2}
$$

Where $E_{s}$ is the energy per information bit of the mapped symbol $X_{m}$. To carry out the threshold test, only the computation of $\sigma_{\hat{X}_{m}}^{2}$ is required, as the soft LLR information is available at the decoder output.

\section{B. OMP Channel Estimation}

The OMP algorithm is a kind of greedy algorithm. It first searches the dictionary for elements that match the received signal, orthogonalizes the selected element, removes the effect of the element from the signal and the dictionary and obtains the signal residual. Then in the remaining dictionary, it continues to search for the element that has the best match with the signal residual, and the above process is repeated until the residual satisfies the set threshold.

Considering that the multipath UWA channel is linearly time invariant for each OFDM block, the channel estimation needs to determine the corresponding delay $\tau_{p}$ for each path. The estimation problem can be reformulated by constructing a socalled dictionary, made of the signals parameterized by a representative selection of possible values of parameter $\tau_{p}$. The path delay $\tau_{p}$ depends on these factors as given by: $\tau_{p} \in\left\{0, \frac{1}{\lambda B}, \frac{2}{\lambda B}, \ldots, \frac{N_{\tau}-1}{\lambda B}\right\}$, where $\lambda$ is the time oversampling factor and there are a total of $N_{\tau}=T_{g} / \lambda B$ delays, where $T_{g}$ is the length of the guard interval. The pilots subcarriers are indexed as the set $S_{p}=\left\{q_{1}, \ldots, q_{K_{p}}\right\}$ with $K_{p}=\left|S_{p}\right|$ pilot subcarriers in total. With these path delays, the dictionary based formulation is given as:

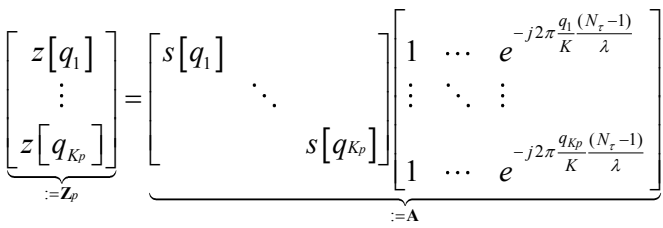

$$
\begin{aligned}
& \underbrace{\left[\begin{array}{l}
\xi_{0} \\
\vdots \\
\xi_{N_{\tau}-1}
\end{array}\right]}_{:=\xi}+\underbrace{\left[\begin{array}{l}
\eta\left[q_{1}\right] \\
\vdots \\
\eta\left[q_{K p}\right]
\end{array}\right]}_{:=\eta}
\end{aligned}
$$

which can be put in vector-matrix form as:

$$
\begin{gathered}
\mathbf{z}_{p}=\left[\mathbf{a}_{0} \cdots \mathbf{a}_{p-1} \cdots \mathbf{a}_{N_{\tau}-1}\right] \boldsymbol{\xi}+\boldsymbol{\eta} \\
\mathbf{z}_{p}=\mathbf{A} \boldsymbol{\xi}+\boldsymbol{\eta}
\end{gathered}
$$

where $\mathbf{a}_{p-1}$ denotes the $p$ th dictionary element and is of size $K_{p} \times 1 ， \mathbf{Z}_{p}$ shows the received pilot information, $\boldsymbol{\eta}$ is the noise vector, $\xi$ shows the channel information to be estimated and $\mathbf{A}$ is the constructed dictionary vector of size $K_{p} \times N_{\tau}$. 
Let $\mathbf{r}_{p}$ be the residual after $p$ iterations with initial value $\mathbf{r}_{0}=\mathbf{z}_{p}$, search for the elements in the dictionary that have the largest inner product of residuals and get the index of the matching element in the dictionary:

$$
S_{p}=\arg \max _{j=1, . ., N_{\tau}-1, j \notin I_{p-1}} \frac{\left|\mathbf{a}_{j}^{H} \mathbf{r}_{p-1}\right|^{2}}{\left\|\mathbf{a}_{j}\right\|_{2}^{2}}
$$

where $I_{p-1}=\left\{s_{1}, s_{2}, \cdots, s_{p-1}\right\}$ is the index of the previous $p-1$ iterations. Schmidt orthogonalization of the selected elements is given by:

$$
\mathbf{u}_{s_{p}}=\mathbf{a}_{s_{p}}-\sum_{i=1}^{p-1} \frac{\left\langle\mathbf{a}_{s_{p}}, \mathbf{u}_{i}\right\rangle}{\left\langle\mathbf{u}_{i}, \mathbf{u}_{i}\right\rangle} \mathbf{u}_{i}
$$

Where $\mathbf{u}_{i}$ is the value of the orthogonalized element chosen for the first time and the estimated values of elements in signal $\xi$ is given by:

$$
\hat{\xi}=\frac{\left\langle\mathbf{u}_{s_{j}}, \mathbf{r}_{p-1}\right\rangle}{\left\|\mathbf{u}_{s_{p}}\right\|_{2}^{2}}
$$

And the residual signal is calculated as:

$$
\mathbf{r}_{p}=\mathbf{r}_{p-1}-\hat{\xi} \mathbf{a}_{s_{p}}
$$

Stop the iterations when $\left\|r_{p}\right\|_{2}^{2}<\varepsilon$ (where $\varepsilon$ is the residual threshold). Finally the channel coefficients at all subcarriers can be constructed as:

$$
\hat{H}[m]=\sum_{p=0}^{N_{\tau}-1} \hat{\xi} e^{-j 2 \pi \frac{m p}{\lambda K}}
$$

\section{LDPC Coding}

Low-density parity-check (LDPC) code is a linear block error correcting code, used for transmitting a message over a noisy transmission channel. Its check matrix is sparse and the codes are capacity-approaching codes, means that the threshold can be set very much close to Shannon capacity limit. The description format of LDPC codes is relatively simple and has strong error correction capability and excellent flexibility, which makes LDPC codes suitable for almost all channels. The amount of computation does not increase dramatically with the increase in code length, therefore keeping the complexity low. There are many design approaches to construct LDPC code check matrix, such as Gallager's construction method, MacKay construction method, repeated accumulation design construction method, - rotation matrix construction method, etc. We used the regular LPDC Gallager codes here with $1 / 2$ code rate and block size of 851 .

\section{RESUlTS}

\section{A. Simulation Results}

A shallow water channel is modeled using Bellhop and the channel impulse response is given in Figure 2, while the simulation parameters for the OFDM system are given in Table 1 below. First of all the iterative and non-iterative receivers are compared, then different feedback methods are compared. The soft feedback method is analyzed for reduced number of pilots and the proposed design is then compared with other soft decision feedback methods.

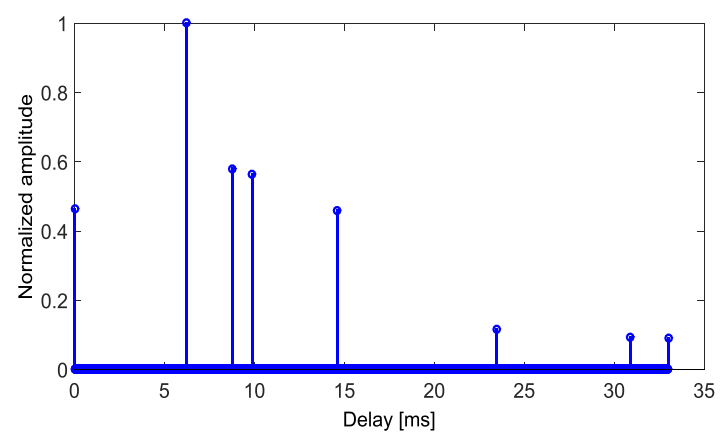

Figure 2: Simulated Channel Impulse response

Table 1

OFDM Simulation Parameters

\begin{tabular}{|c|c|c|}
\hline Serial \# & Parameter & Value \\
\hline 01 & Sampling frequency & $48 \mathrm{kHz}$ \\
\hline 02 & Communication bandwidth & $6 \mathrm{kHz}-12 \mathrm{kHz}$ \\
\hline 03 & Total number of subcarriers & 1024 \\
\hline 04 & Number of data carriers & 851 \\
\hline 05 & Number of pilots & 125 \\
\hline 06 & Number of Null carriers & 48 \\
\hline 07 & OFDM symbol period & $170.67 \mathrm{~ms}$ \\
\hline 08 & Cyclic prefix length & $40 \mathrm{~ms}$ \\
\hline 09 & Spectrum utilization & $0.67 \mathrm{~b} / \mathrm{s} / \mathrm{Hz}$ \\
\hline 10 & Communication rate & $4.04 \mathrm{~kb} / \mathrm{s}$ \\
\hline
\end{tabular}

The performance comparison is done by comparing the BER performance as well as normalized mean square error (NMSE) performance. The NMSE is defined as:

$$
\mathrm{NMSE}=E\left(\|\mathbf{H}-\hat{\mathbf{H}}\|^{2} /\|\mathbf{H}\|^{2}\right)
$$

First of all we compare the BER and NMSE of the iterative receiver with non-iterative receiver in Figure 3. The noniterative receiver is similar to the iterative receiver with single iteration. The performance of iterative receiver even after the second iteration is far better than that of non-iterative receiver We use soft information feedback in our design, as the overall 
performance of soft-decision feedback is better than that of hard-decision feedback, because soft information feedback can generally make more use of symbol statistics than hard information feedback $[14,15]$.

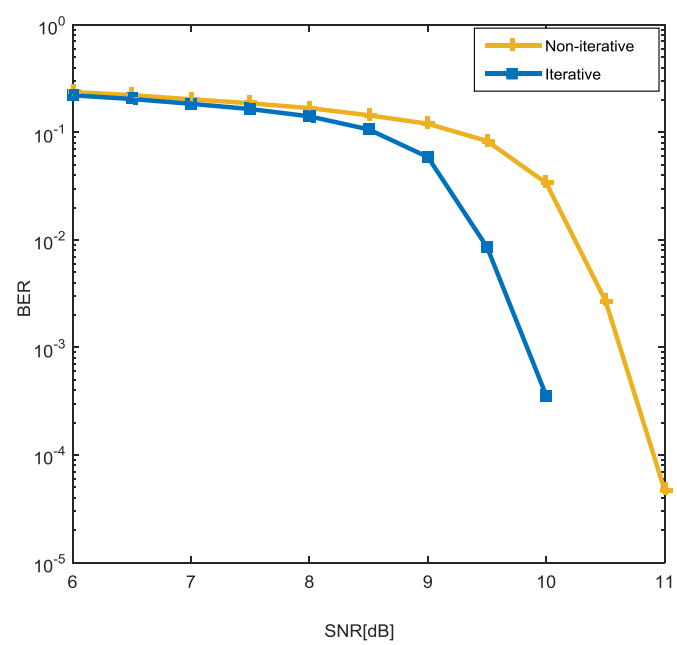

a) BER Vs SNR

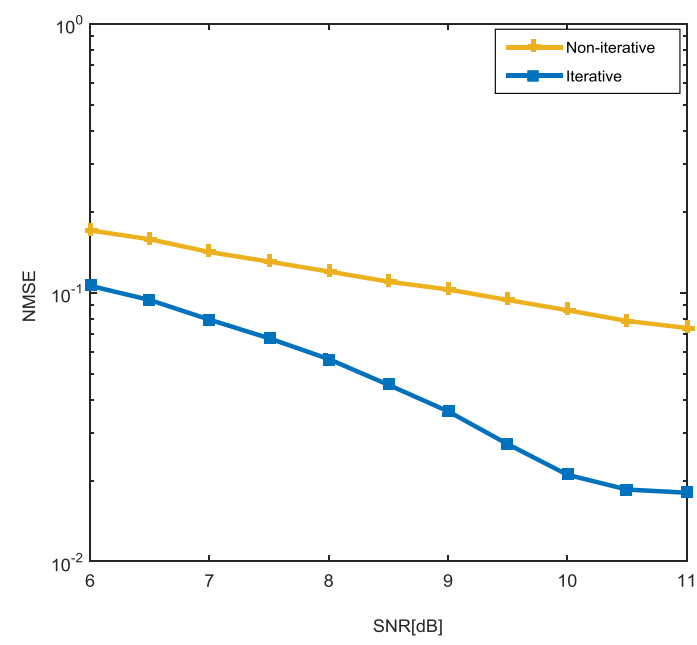

b) NMSE Vs SNR

Figure 3: Iterative Vs Non-iterative receiver

Next, we compare the performance of iterative channel estimation at different pilot intervals by changing the pilot interval from 4 to 8 and 12 while using soft decision feedback method with 4 iterations, as shown in Figure 4 . The curves in figure 4 (a), (b) and (c) show the comparison between iterative and non-iterative channel estimation for the pilot intervals 12 , 8 and 4 respectively. It can be seen that when the pilot interval increases, the channel estimation performance decreases, i.e., smaller number of pilots cause a decrease in channel estimation performance. Furthermore, when the pilot interval is large, the performance gap between the iterative and noniterative channel estimations is more obvious, whereas, the difference is less obvious in case of smaller pilot interval. This shows that the additional pilot information feedback when the number of pilots is small is more important for the channel estimation.

In the Figure 4(d), the comparison of pilot spacing 4, 8 and 12 shows a significant difference in the performance of the iterative channel estimation and the performance degrades for the larger pilot interval. This Figure 4 further shows that the performance of pilot spacing 4 and 8 is very close in case of iterative channel estimation, which shows that the use of iterative method can easily reduce the number of pilots used.
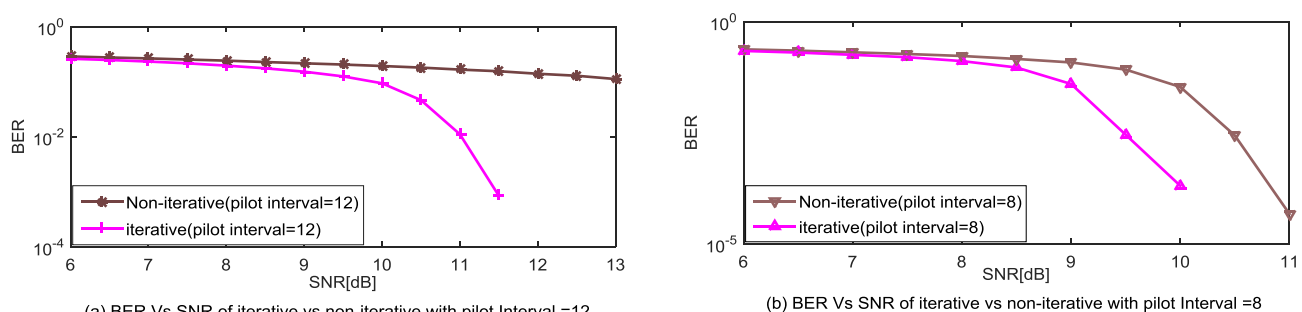

(a) BER Vs SNR of iterative vs non-iterative with pilot Interval $=12$
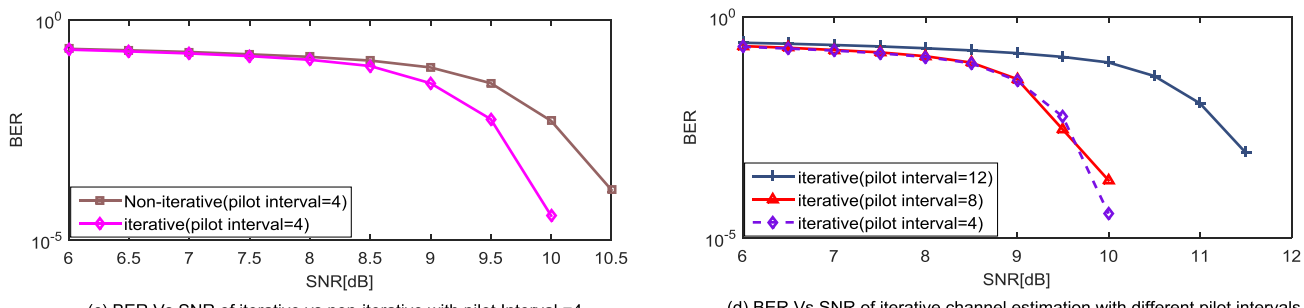

(c) BER Vs SNR of iterative vs non-iterative with pilot Interval $=4$

Figure 4: Comparison of Iterative Channel Estimation Performance at different Pilot intervals using Soft feedback method 

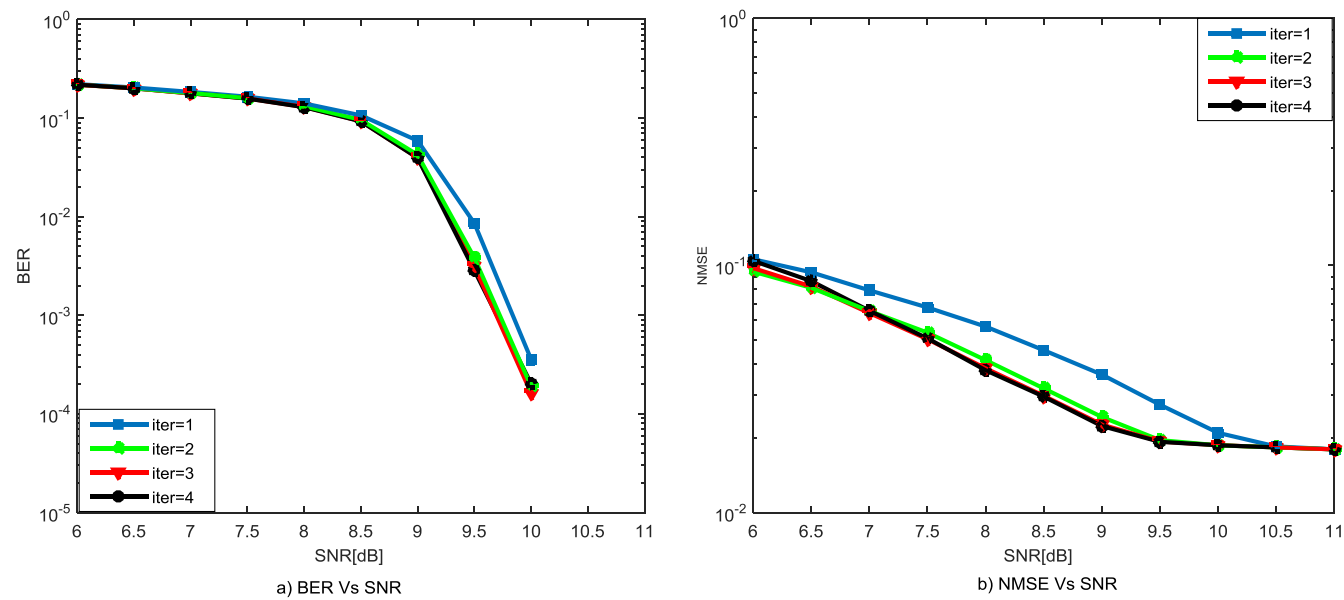

Figure 5: Performance Comparison of cost based soft feedback method at different number of iterations

Figure 5 compares the BER and NMSE the performances of the proposed feedback method based on the cost function for different number of iterations. It can be seen from the figure that system's BER and NMSE is significantly reduced with the increase in number of iterations and the system's performance is gradually improved until it reaches stability.

Next we compare the BER performance of the proposed feedback method with soft decision feedback for every iteration as shown in Figure 6. It can be seen that the performance of the proposed method is better than the soft decision feedback in each iteration. The performance gap decreases with the increase in iterations as can be seen in the figures that the gap is more in the first iteration and is reduced in the fourth iteration when the system stabilizes, however the performance of soft feedback based on cost function is still better than soft feedback method. It is also concluded that as the proposed method performs better even in the first and second iterations, the processing time and complexity can be reduced if we reduce the number of iterations for this method.
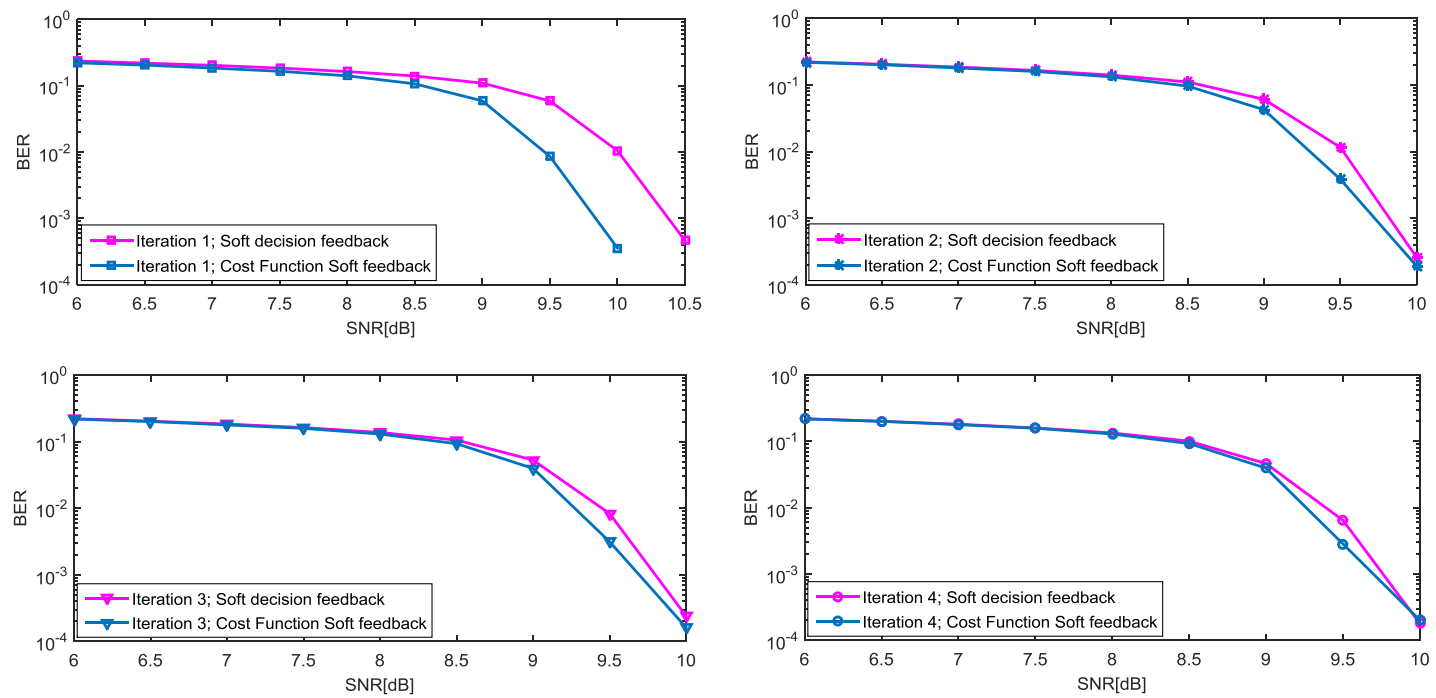

Figure 6: Comparison of soft decision feedback with cost based soft decision feedback

\section{B. Experimental Results}

The performance of the proposed iterative receiver algorithm in underwater acoustic SISO OFDM communication system is verified via sea trials. The experimental data was collected in experiments conducted in
South China sea. The OFDM system experimental parameters are shown in Table 2. The depth of sea water was 60 to 70 meters with good sea conditions. Two ships were used to verify the communication performance. The receiving vessel was anchored and four hydrophones were deployed $30 \mathrm{~m}$ deep; the transmitter was deployed $27 \mathrm{~m}$ below the launching ship. 
The launching ship was moving towards the receiving ship at a speed of 2 knots and kept moving from a position $3 \mathrm{~km}$ away to $1 \mathrm{~km}$ and was continuously sending test signals.

Table 2

Sea Experiment OFDM System Parameters

\begin{tabular}{|l|l|l|}
\hline Serial \# & Parameter & Value \\
\hline 01 & Sampling frequency & $48 \mathrm{kHz}$ \\
\hline 02 & Communication bandwidth & $6 \mathrm{kHz}-10 \mathrm{kHz}$ \\
\hline 03 & Total number of subcarriers per transmitter & 681 \\
\hline 04 & Number of data carriers per transmitter & 595 \\
\hline 05 & The number of pilots at each transmitter & 86 \\
\hline 06 & OFDM symbol period & $170.25 \mathrm{~ms}$ \\
\hline 07 & Cyclic prefix length & $20 \mathrm{~ms}$ \\
\hline 08 & Cyclic Suffix Length & $5 \mathrm{~ms}$ \\
\hline 09 & Spectrum utilization & $0.76 \mathrm{~b} / \mathrm{s} / \mathrm{Hz}$ \\
\hline 10 & Communication rate & $3.05 \mathrm{~kb} / \mathrm{s}$ \\
\hline
\end{tabular}

Each frame of data contains 8 OFDM symbols, while QPSK mapping is used. Two encoding methods are used: $1 / 2$ code rate convolutional code and $1 / 2$ code rate LDPC, both with the same information sequence length.

The performance of the convolutional code and LDPC code, and the performance of the LS channel estimation algorithm and the OMP algorithm are compared and analyzed. The signals of the second receiver and fourth receiver are processed respectively, as shown in Figure 7.

Comparing the two upper curves in Figure 7 (a) \& (b), it can be observed that with the same channel coding scheme, the performance of OMP channel estimation is better than the traditional LS channel estimation. Comparing the second and third curves, we can see that when the same channel estimation algorithm is used, the LDPC code performs better than the convolutional code. Therefore it is concluded that a combination of LDPC code and OMP channel estimation gives the best performance.

Next we verify the performance of the iterative reception algorithm. Taking 7 frames of data from the hydrophone 4 for the analysis. The number of bits transmitted in each frame of data is $595 \times 8 \times 2=9520$, as there are 8 OFDM symbols in each frame and each symbol has 595 data carriers, while the modulation is QPSK so there are two bits in each symbol. Table 3 lists the number of error bits for different data frames at different iterations.

It can be observed from Table 3 that the performance of different data frames after receiver's initial processing (without iterations) is quite different. The relative movements of the transmitter and receiver during the experiment and the different interference of frames with the background noise and the channel conditions can be the reasons for these large differences. Let us take the first frame and the fourth frame of the received data that are widely different in performance, as an example for analysis.

Figure 8 (a) \&(b) shows the data signal received for these two frames, normalization is performed and the frame header LFM signal is used to measure the channel impulse response experienced by the two frames of data, as shown in Figure 9. It can be seen that the data of the first frame is more affected by noise than the data of the fourth frame, furthermore the experienced channel for the first frame is more complicated than the fourth frame. This is the reason why the bit error rate of the first frame data is high.
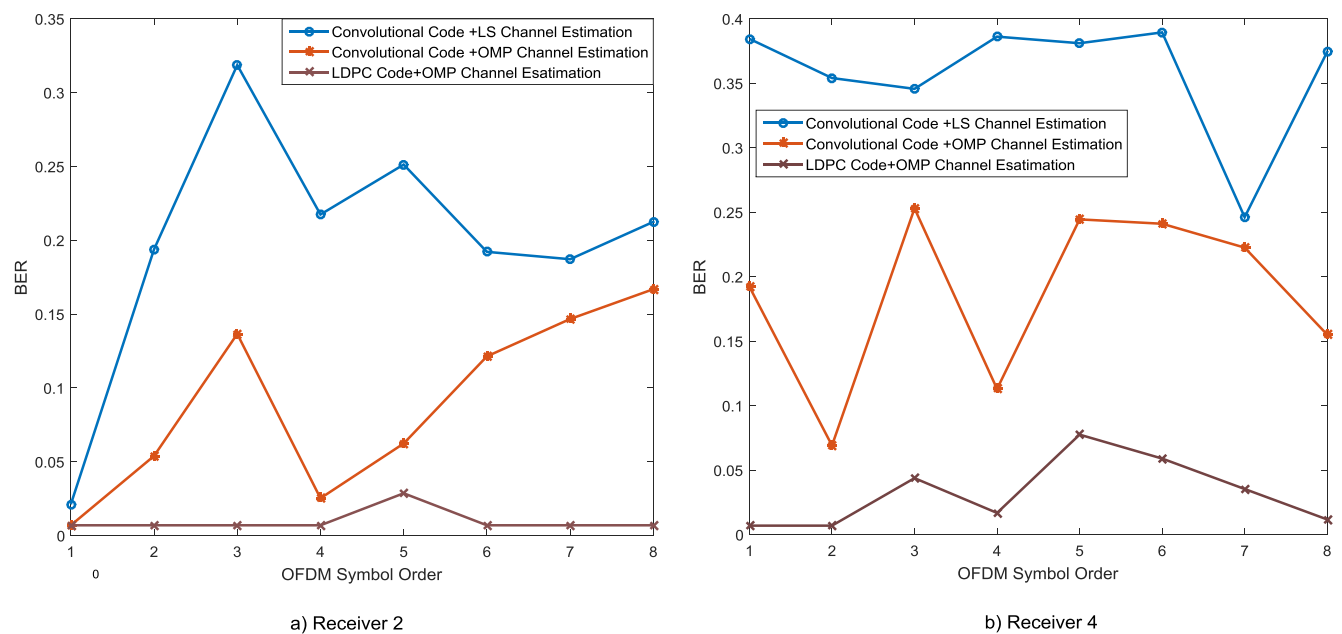

Figure 7: Comparison of different channel estimation methods with different channel coding schemes 
Cost Function based Soft Feedback Iterative Channel

Estimation in OFDM Underwater Acoustic Communication

Table 3

Statistics of the number of bit errors at different iteration times for different data frames

\begin{tabular}{|c|c|c|c|c|c|c|}
\hline Frame No. & No iteration & $1^{\text {st }}$ Iteration & $2^{\text {nd }}$ Iteration & $3^{\text {rd }}$ Iteration & $4^{\text {th }}$ Iteration & $5^{\text {th }}$ Iteration \\
\hline 01 & 1086 & 526 & 270 & 162 & 36 & 0 \\
\hline 02 & 512 & 228 & 48 & 0 & 0 & 0 \\
\hline 03 & 881 & 346 & 150 & 4 & 0 & 0 \\
\hline 04 & 0 & 0 & 0 & 0 & 0 & 0 \\
\hline 05 & 116 & 0 & 0 & 0 & 0 & 0 \\
\hline 06 & 184 & 0 & 0 & 0 & 0 & 0 \\
\hline 07 & 2146 & 2146 & 2262 & 2476 & 2684 & 2904 \\
\hline
\end{tabular}

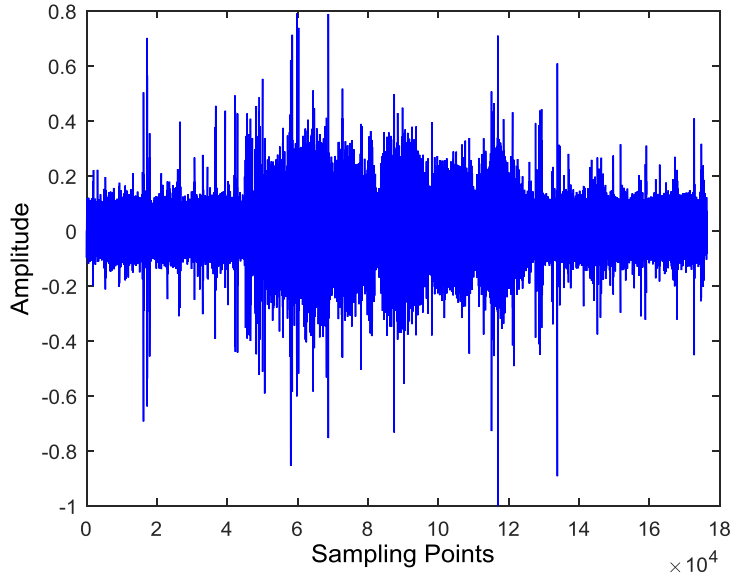

a) Data Frame 1

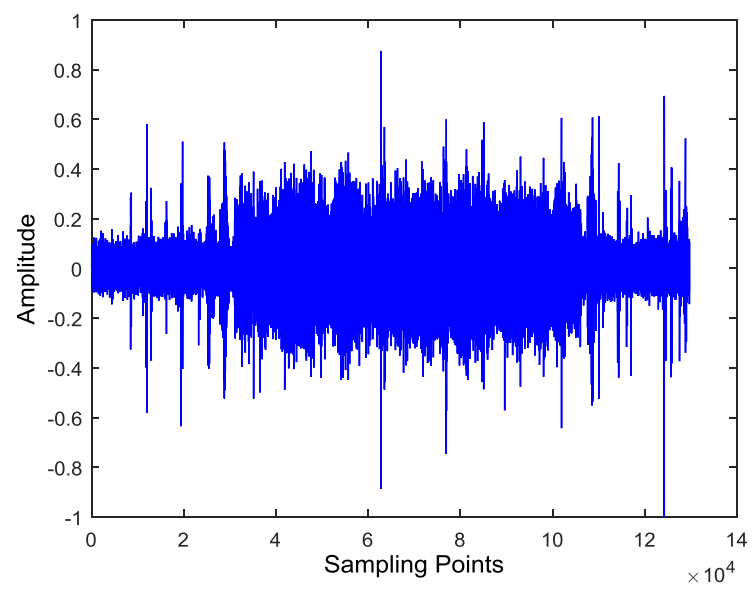

b) Data Frame 4

Figure 8 : Received signal in the sea experiment

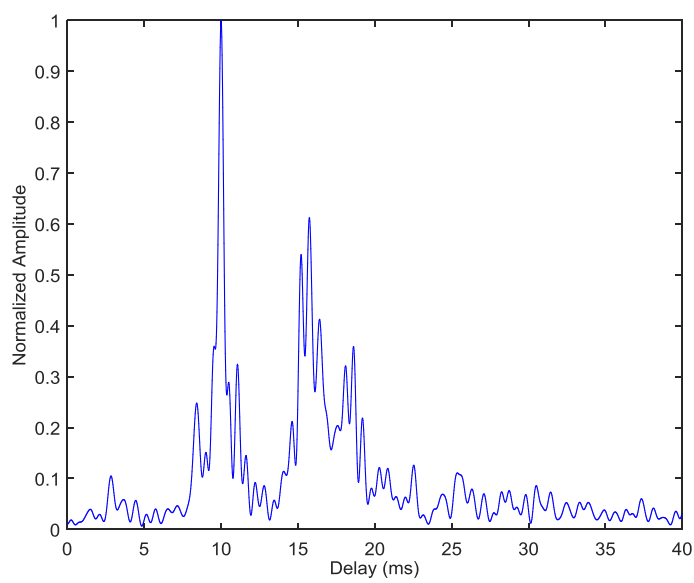

a) Channel experienced by Data Frame 1

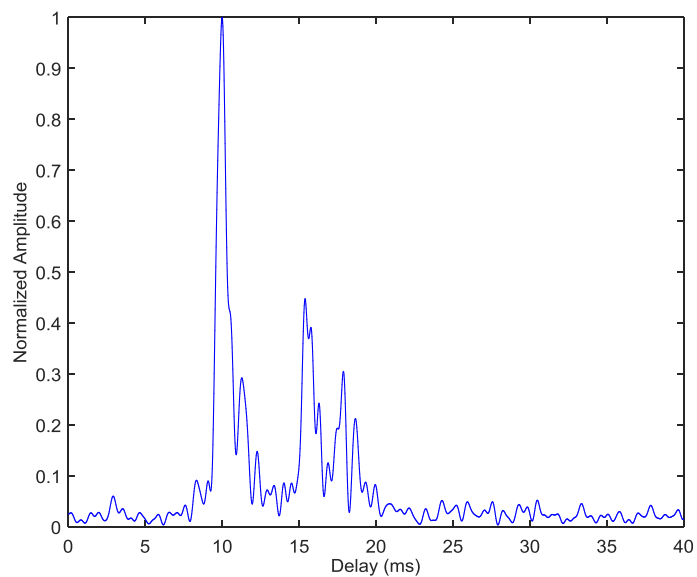

b) Channel experienced by Data Frame 4

Figure 9: Measurement of CIRs in sea experiment

Further analysis of table 3 shows that with the increase in the number of iterations, the number of errors in the decoded bits gradually reduces and with each increase in iteration, the number of erroneous bits almost halves. If the number of erroneous bits is more, it needs multiple iterations to reduce this number to 0 as obvious for the first frame, whereas the number of the initial erroneous bits of the 5th and 6th frames is less; therefore the number of erroneous bits is reduced to 0 right after the first iteration.

The effectiveness of the iterative reception algorithm is 
verified, however the iterative reception algorithm also has its limitations. For the case where the initial bit error rate is very high, such as the seventh frame (the number of error bits is 2146, the corresponding bit error rate is about 0.23 ), the number of bit errors increases after the iteration instead. The reason is that there may exist some error propagation in the iterative process and the processing capability of the iterative reception algorithm has a certain range or threshold. Just like the error correction code, it may be not be effective after a certain error correction threshold is exceeded.

\section{CONCLUSION}

This paper proposes a receiver based on cost function threshold driven soft decision feedback iterative channel estimation technique for OFDM UWA communication. The receiver exploits OMP channel estimation and LDPC coding schemes. The performance of the proposed receiver is verified by simulations as well as sea experiments. The proposed receiver is compared with other non-iterative and soft and hard decision feedback iterative receivers and it outperforms them in terms of BER and NMSE performance. During the sea trials, combinations of different channel estimation schemes and coding schemes are compared and LDPC coding with OMP channel estimation outperformed the LS estimation and convolutional codes. Furthermore the better performance of the proposed receiver is proved in the sea experiment.

\section{REFERENCES}

[1] Mandal, A. and R. Mishra, Design of Pipelined Adaptive DFE Architecture For High Speed Channel Equalization INFOCOMMUNICATIONS JOURNAL, 2015. 7(3).

[2] Horvath, B. and P. Horvath, Establishing Lower Bounds on the Peakto-Average-Power Ratio in Filter Bank Multicarrier Systems. INFOCOMMUNICATIONS JOURNAL, 2015. 7(3).

[3] Qiao, G., et al., MIMO-OFDM underwater acoustic communication systems - A review. Physical Communication, 2017. 23: p. 56-64.

[4] Li, B., et al. MIMO-OFDM Over An Underwater Acoustic Channel. in OCEANS 2007. 2007.

[5] Stojanovic, M. Low Complexity OFDM Detector for Underwater Acoustic Channels. in OCEANS 2006. 2006.

[6] Carrascosa, P.C. and M. Stojanovic. Adaptive MIMO detection of OFDM signals in an underwater acoustic channel. in OCEANS '08. 2008.

[7] Stojanovic, M. OFDM for underwater acoustic communications: Adaptive synchronization and sparse channel estimation. in 2008 IEEE International Conference on Acoustics, Speech and Signal Processing. 2008.

[8] Stojanovic, M. Adaptive Channel Estimation for Underwater Acoustic MIMO OFDM Systems. in Digital Signal Processing Workshop and 5th IEEE Signal Processing Education Workshop, 2009. DSP/SPE 2009. IEEE 13th. 2009.

[9] Huang, J., et al. Iterative sparse channel estimation and decoding for underwater MIMO-OFDM. in OCEANS 2009. 2009

[10] Huang, J., C.R. Berger, and S. Zhou. Comparison of basis pursuit algorithms for sparse channel estimation in underwater acoustic OFDM. in OCEANS 2010 IEEE - Sydney. 2010.

[11] Upadhyay, N., M. Tiwari, and J. Singh, LDPC Based MIMO-OFDM System for Shallow Water Communication using BPSK. International Journal of Electronics \& Communication Technology (IJECT) 2015. Vol. 6 (Issue 4).

[12] Niu, H. and J.A. Ritcey. Iterative channel estimation and decoding of pilot symbol assisted LDPC coded QAM over flat fading channels. in The Thrity-Seventh Asilomar Conference on Signals, Systems \& Computers, 2003. 2003.
[13] Valenti, M.C. and B.D. Woerner, Iterative channel estimation and decoding of pilot symbol assisted turbo codes over flat-fading channels. IEEE Journal on Selected Areas in Communications, 2001. 19(9): p. 1697-1705.

[14] Laot, C., A. Glavieux, and J. Labat, Turbo equalization: adaptive equalization and channel decoding jointly optimized. IEEE Journal on Selected Areas in Communications, 2001. 19(9): p. 1744-1752.

[15] Auer, G. and J. Bonnet. Threshold Controlled Iterative Channel Estimation for Coded OFDM. in 2007 IEEE 65th Vehicular Technology Conference - VTC2007-Spring. 2007.

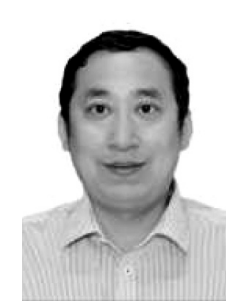

Gang Qiao received the B.S., M.S., and Ph.D. degrees in underwater acoustic engineering from the Harbin Engineering University (HEU), Harbin, China, in 1996, 1999, and 2004, respectively. He visited the Department of Electrical Engineering, University of Washington, Seattle, WA, USA, as a Senior Visiting Scholar in 2015. He has been a full Professor with HEU since 2007. His research interests lie in the areas of underwater acoustic communication and networking, and underwater acoustic target detection and localization.

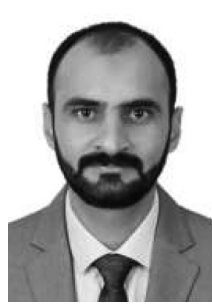

Zeeshan Babar received the B.S. and M.S. degrees from National University of Science and Technology (NUST) and University of Engineering and Technology Taxila Pakistan in 2008 and 2012 respectively. Currently he is pursuing his Ph.D. in Underwater Acoustic Engineering from Harbin Engineering University (HEU), Harbin, China. His research interests lie in the areas of underwater acoustic communication and networking.

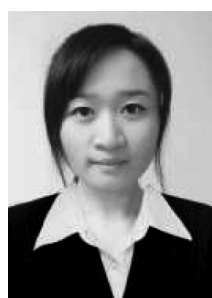

Lu Ma received the B.S. and Ph.D. degrees in signal and information processing from the Harbin Engineering University (HEU), Harbin, China, in 2010 and 2016, respectively. She visited the University of Connecticut, Storrs, CT, USA, from October 2014 to October 2015. She has been an Assistant Professor with the College of Underwater Acoustic Engineering, HEU, since November 2016. Her research interests lie in the areas of multicarrier and multiuser communications for underwater acoustic channels.

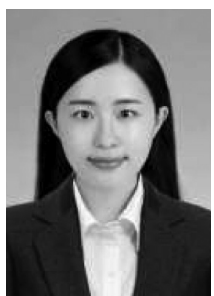

Xue Li received her B.S. and M.S. degrees in 2015 and 2018 respectively from Harbin Engineering University, Harbin, China and is currently involved in active research in the field of underwater acoustics. Her research interests lie in the areas of underwater acoustic communication andnetworking. 